\title{
Early cerebrovascular ultrasonography as a predictor of hemorrhagic transformation after thrombectomy
}

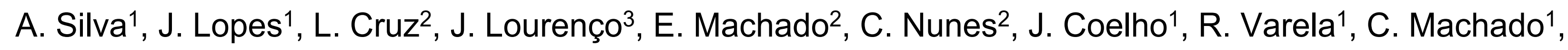

B. Rodrigues ${ }^{1}$, C. Nunes ${ }^{1}$, C. Macário ${ }^{1}$, L. Cunha ${ }^{1}$, G. Cordeiro ${ }^{1}$, F. Silva ${ }^{1}$, J. Sargento-Freitas ${ }^{1}$.

${ }^{1}$ Coimbra University and Hospital Centre, Neurology Department, Coimbra, Portugal. ${ }^{2}$ Coimbra University and Hospital Centre, Neuroradiology Department, Coimbra, Portugal. ${ }^{3}$ Coimbra University and Hospital Centre, Internal Medicine Department, Coimbra, Portugal

\section{Background and Aims}

Acute recanalization of a large vessel occlusion has a dramatic clinical impact in functional recovery. However, it increases the possibility of reperfusion lesion and hemorrhagic transformation. Reperfusion injury is initially a hemodynamic phenomenon, which through its persistency might increase tissue lesion. Transcranial Color Doppler (TCCD) might be a useful tool for early diagnosis, though its diagnostic acuity has not yet been assessed.

\section{Method}

Retrospective cohort study including consecutive patients from a 33 month period with large vessel occlusion who achieved arterial recanalization after thrombectomy (final $\mathrm{TICl} 2 \mathrm{~b}$ or 3 ) and had TCCD in the first 24 hours. Parameters measured were mean velocity from M1 segment of symptomatic and asymptomatic MCA and symptomatic/asymptomatic MCA ratio (RaMCA). Hemorrhagic transformation was quantified by Head CTScan at 24 hours.

\section{Results}

We included 101 patients, mean age of 67.99 years (SD:13.86), being $59.00(58.40 \%)$ men.

\begin{tabular}{|l|l|}
\hline \multicolumn{2}{|l|}{ Table 1. Characteristics of Study Population } \\
\hline Age (years), mean (SD) & $6.99(13.86)$ \\
\hline Male, $\mathrm{n}(\%)$ & $59.00(58.40 \%)$ \\
\hline
\end{tabular}

Mean velocity on symptomatic MCA was $59.51 \mathrm{~cm} / \mathrm{sec}$ (SD:20.21), while mean RaMCA was 1.06 (SD: 0.36). Mean velocity for symptomatic MCA was not statistically different on patients who showed hemorrhagic transformation: $66.43 \mathrm{~cm} / \mathrm{sec}$ (SD: 11.17 ) vs. $58.78 \mathrm{~cm} / \mathrm{sec}$ (SD: 20.93), $p=0.072$.

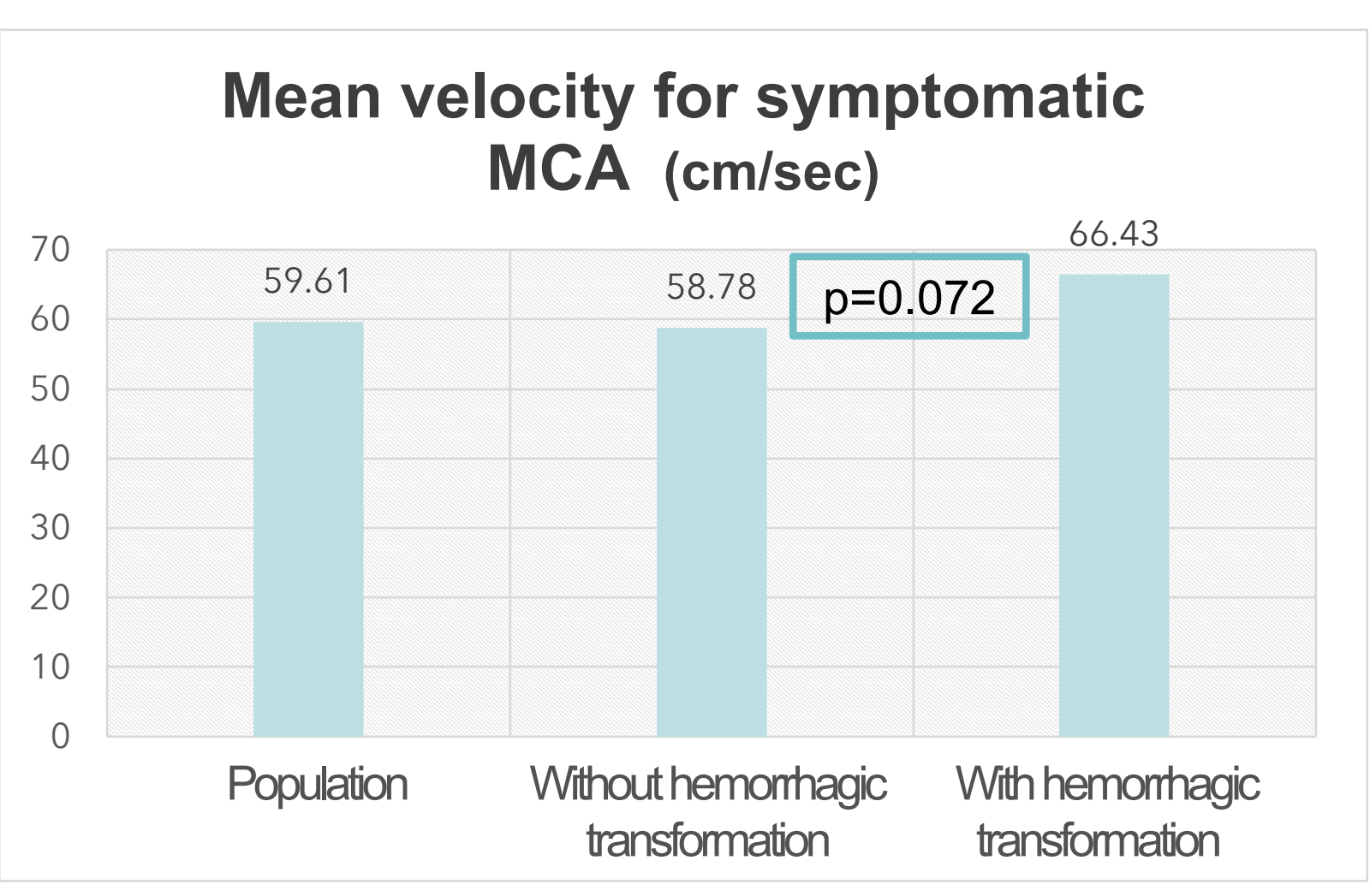

Graph 1. Mean velocity for symptomatic MCA

RaMCA was higher in the cohort of patients with hemorrhagic transformation: 1.32 (SD:0.39) vs. 1.02 (SD: $0.34), p=0.01$. In a multivariable analysis, RaMCA remained an independent predictor of hemorrhagic transformation: OR: $6.84(1.19-39.17) p=0.031$.

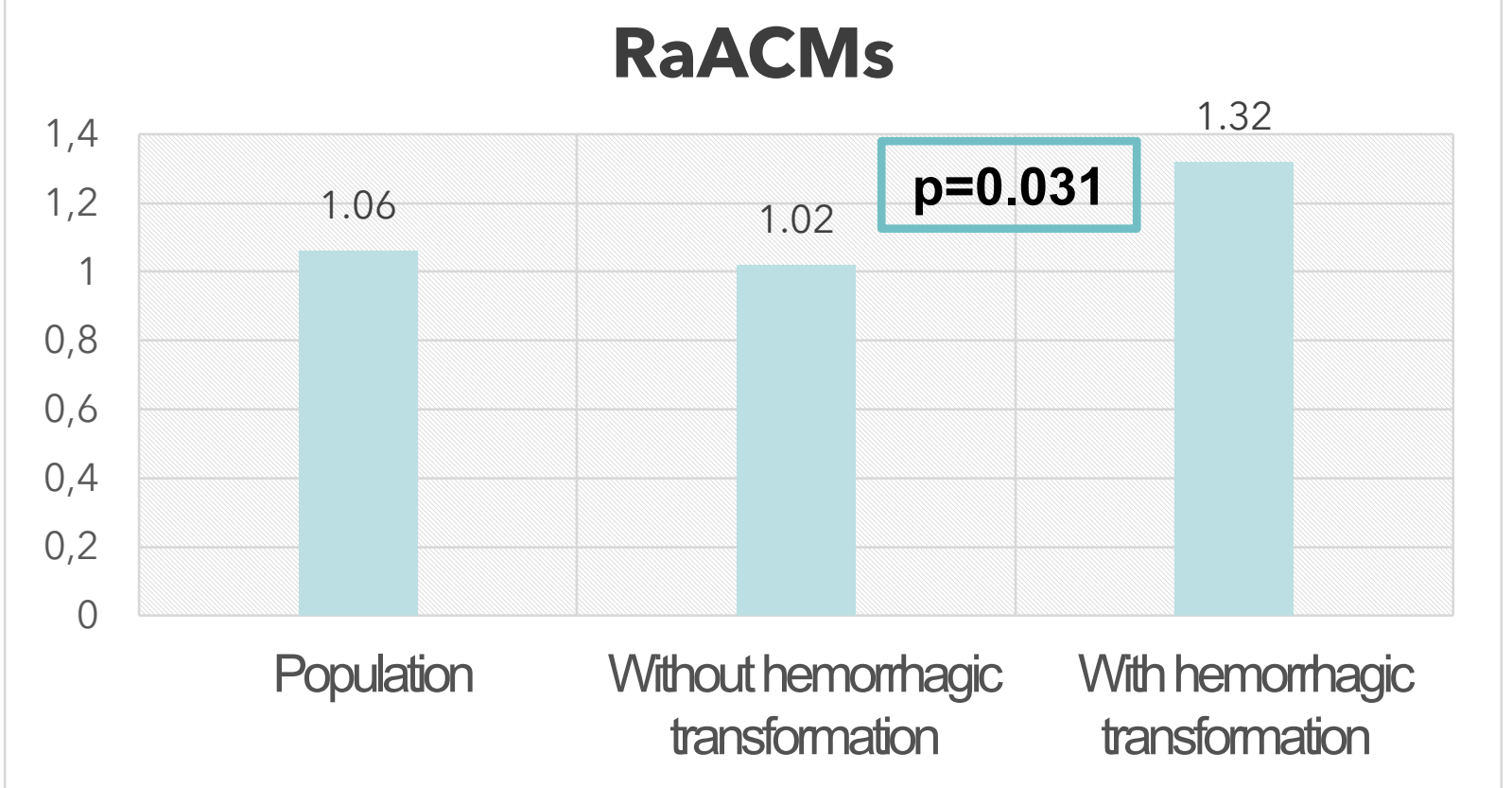

Graph 2. Symptomatic/asymptomatic MCA ratio (RaMCA).

\section{Conclusion}

RaMCA evaluated through TCCD in the first 24 hours after anterior circulation ischemic stroke in patients who achieve recanalization after thrombectomy might be an indepedent predictor of hemorrhagic tranformation.

\section{Bibliography}

Sarkar S., et al. Role of transcranial Doppler ultrasonography in stroke. Postgrad Med J. 2007 Nov, 83(985):683-689.

D'Andrea A, et al. Transcranial Doppler ultrasonography. From methodology to major dinical applications. World J Cardiol. 2016 Jul 26; 8(7):383-400.

Naqvi J., et al. Transcranial Doppler Uttrasound: A Review of the Physical Principles and Major Applications in Critical Care. Int J Vasc Med. 2013; 2013: 629378. 